\title{
La madre de las clasificaciones: términos de parentesco en la categorización nominal en toba (guaycurú)
}

\author{
The Mother of Classification: \\ Kinship Terms in Noun Categorization in Toba (Guaycuruan) \\ Paola Cúneo ${ }^{1}$
}

\begin{abstract}
Resumen
El objetivo de este artículo es profundizar en el estudio de los morfemas de parentesco 'madre' y 'padre' en toba explorando su función específica en la clasificación de nombres. Los morfemas de parentesco en esta lengua constituyen un rico y complejo tema de estudio y han despertado el interés de estudios tanto lingüísticos como antropológicos y etnobiológicos.

El trabajo se propone precisar los aspectos formales, semánticos y funcionales de los morfemas léxicos late?e 'madre' y lta?a 'padre'. Mostraremos que los mismos participan de manera productiva en la formación de nombres compuestos y poseen una función destacada no solo en la identificación de entidades sino también en la conformación de dominios léxicos. A su vez, evidenciamos que, además de su función derivacional, participan en la categorización de los nombres. La complejidad de su significado radica en que estas formas codifican las nociones de semejanza, aproximación y no-prototipicalidad junto a la de aumentativo.

Palabras-clave: Toba. Clasificación nominal. Composición. Términos de parentesco. Términos de clase.
\end{abstract}

\section{Abstract}

The aim of this paper is to delve into the study of kinship morphemes for "mother" and "father" in the Toba language by examining their specific role in the noun classification. Kinship morphemes in this language are a rich and complex field of study, as they have awakened the interest of both linguists and anthropologists.

The paper intends to specify the formal, semantic and functional aspects of lexical morphemes late?e 'mother,' and lta?a 'father', in the Toba language. We will show their productive involvement in the formation of compound nouns, and major role not only in the identification of entities but also in the configuration of lexical domains. In addition, we have observed that, besides their derivational function, they are involved in the categorization of nouns. The complexity of their meanings lies in the fact that these forms encode notions of similarity, approximation and no-prototipicality, as well as the augmentative.

Keywords: Toba. Nominal classification. Noun compounding. Kinship terms. Class terms.

${ }^{1}$ Doctora de la Universidad de Buenos Aires (Lingüística). Investigadora del Consejo Nacional de Investigaciones Científicas y Técnicas (CONICET) y docente de la Universidad de Buenos Aires. pcuneo@gmail.com 


\title{
1. Introducción
}

Entre los modos en los que las lenguas indígenas sudamericanas clasifican los nombres y sus referentes, han recibido comparativamente poca atención en la literatura aquellos sistemas clasificatorios que poseen funciones de derivación y que generalmente expanden el vocabulario de la lengua. ${ }^{2} \mathrm{~A}$ su vez, la discusión sobre las tendencias translingüísticas de gramaticalización y extensión metafórica que transitan los términos de relación que pertenecen al vocabulario básico de las lenguas -como, padre, madre, hijo-es otro de los temas de estudio que enmarcan este trabajo (cf. Matisoff 1992; Heine y Kuteva 2002). ${ }^{3}$

El objetivo de este artículo es profundizar en el estudio de los morfemas léxicos de parentesco late?e 'su madre' y lta?a 'su padre' en toba, a partir de descripciones previas (Cúneo 2012, 2013c), y explorar su función específica en la clasificación de nombres. Mostraremos que estas formas participan de manera productiva en la formación de compuestos nominales y poseen una función destacada tanto en la identificación de entidades como en la conformación de dominios léxicos. A su vez, evidenciamos que, además de su función derivacional, participan en la categorización de los nombres. La complejidad de su significado radica en que estas formas codifican las nociones de semejanza y aproximación junto a la de aumentativo. Los siguientes son ejemplos representativos del uso de los morfemas de padre y madre:

(1)a. $q o y o^{4}$

'ave, pájaro'

\begin{abstract}
${ }^{2}$ Una excepción muy motivadora para este trabajo es Facundes (2009) para la lengua apurinã (arawak). Cf. Aikhenvald (2000:266-8) -y las referencias allí citadas- para una síntesis de la interacción entre diferentes sistemas de clasificación nominal y mecanismos de derivación.

${ }^{3}$ El artículo de Matisoff "The Mother of All Morphemes. Augmentatives and diminutives in areal and universal perspective" inspiró el título del presente trabajo. Considero aquí las formas 'madre' (FEM) y 'padre' (MASC) como parte del mismo caso de estudio. Matisoff incluye también las formas para 'hijo'.

${ }^{4} \mathrm{El}$ inventario fonológico de la lengua y las correspondencias ortográficas que utilizo en este trabajo, se consignan en la tabla 1.
\end{abstract}

Tabla 1. Inventario fonológico toba (basado en Messineo 2003: 36)

[y correspondencias ortográficas utilizadas]:

\begin{tabular}{|l|l|l|l|l|l|l|l|l|l|l|l|l|}
\hline & \multicolumn{2}{l}{ labial } & \multicolumn{2}{l|}{ alveolar } & \multicolumn{2}{l|}{ palatal } & \multicolumn{2}{l|}{ velar } & \multicolumn{2}{|l|}{ uvular } & \multicolumn{2}{|l|}{ glotal } \\
\hline oclusiva & $\mathrm{p}$ & & $\mathrm{t}$ & $\mathrm{d}$ & $\mathrm{t} \int[\mathrm{ch}]$ & & $\mathrm{k}$ & $\mathrm{g}$ & $\mathrm{q}$ & $\mathrm{g}$ & $\mathrm{?}$ & \\
\hline fricativa & & & $\mathrm{s}$ & & $\int[\mathrm{sh}]$ & $\mathrm{3}[\mathrm{z}]$ & & & & & & \\
\hline nasal & & $\mathrm{m}$ & & $\mathrm{n}$ & & $\mathrm{n}[\tilde{\mathrm{n}}]$ & & & & & & \\
\hline tap & & & & $\mathrm{r}[\mathrm{r} / \mathrm{d}]$ & & & & & & & & \\
\hline lateral & & & & 1 & & $\kappa$ & & & & & & \\
\hline glide & & $\mathrm{w}$ & & & & $\mathrm{y}$ & & & & & & \\
\hline
\end{tabular}


b. qoyo-lta?a

ave-TC.MASC ${ }^{5}$

'reinamora (variedad de ave)' ?Cyanocompsa brissonii

(2)a. potay

'oso hormiguero' Myrmecophaga tridactyla

b. potay-late?e

oso.hormiguero-TC.FEM

'oso melero' Tamandua tetradactyla (de menor tamaño que potay)

(3)a. l-yaGaata

Pos3-dedo

'su dedo (de la mano)'

b. l-yaGaata-late?e

POS3-dedo-TC.FEM

'su pulgar'

En toba, las formas que expresan relaciones nucleares parentales constituyen un rico y complejo tema de estudio dado que han despertado el interés de estudios tanto lingüísticos como antropológicos y etnobiológicos, que se preguntan cómo interpretar y cómo traducir estas formas. En trabajos previos, las hemos considerado entre las formas de aumentativo (Cúneo 2013b) y como parte de un conjunto de términos "especializados" que conforman nombres de plantas y animales (Cúneo 2012). ${ }^{6}$ Además, su uso y su significado despiertan un interés etnográfico dado que constituyen los nombres de los "dueños" de las especies animales, que entablan y regulan las relaciones entre los seres.

En este trabajo nos proponemos precisar los aspectos formales, semánticos y funcionales de los morfemas de parentesco en toba.

\begin{tabular}{|c|c|c|}
\hline \multicolumn{3}{|c|}{ vocales } \\
\hline $\mathrm{i}$ & & $\mathrm{o}$ \\
\hline $\mathrm{e}$ & & \\
\hline & $\mathrm{a}$ & \\
\hline
\end{tabular}

${ }^{5}$ Las abreviaturas utilizadas para las glosas son las siguientes: COMP (compuesto nominal); DD (determinante demostrativo); FEM (género femenino); FN (frase nominal); MASC (género masculino); N (nombre); NMZ (nominalizador); PL (plural); POS (marcador de poseedor); TC (término de clase); 3 (tercera persona). Las referencias científicas botánicas y zoológicas corresponden a Martínez (2009) y a Medrano et al. (2011), respectivamente.

${ }^{6}$ Algunos de los ejemplos que presentamos aquí fueron introducidos anteriormente en Messineo y Cúneo (2011) y Cúneo (2012, 2013a, b). 
La lengua toba (qom liaqtaqa) pertenece a la familia lingüística guaycurú, junto con el pilagá, el mocoví y el caduveo, y las ya extintas abipón y mbayá. El grupo toba está compuesto por aproximadamente 70.000 personas, ${ }^{7}$ que habitan mayoritariamente en la región del Gran Chaco, en las provincias argentinas de Chaco, Formosa y Salta, en el sudeste de Bolivia y en las cercanías de la localidad de El Cerrito en Paraguay. Como consecuencia de procesos migratorios, los tobas viven también en asentamientos permanentes próximos a las grandes ciudades (Resistencia, Pte. R. Sáenz Peña, Rosario, Santa Fe, Buenos Aires y La Plata, en Argentina). Desde el punto de vista sociolingüístico, el toba es una lengua amenazada dado que está siendo desplazada por el español en ciertos ámbitos comunicativos -especialmente en los contextos urbanos- y, además, porque sus hablantes sufren de marginación social y económica.

Los principales estudios previos sobre la lengua toba pueden encontrarse en Censabella (2002) y Messineo (2003), y los recursos de formación de palabras y clasificación nominal han constituido el foco de mi investigación doctoral (Cúneo 2013a).

Algunas de las características tipológicas de la lengua toba relevantes para el presente trabajo son las siguientes. En primer lugar, el toba se caracteriza por su complejidad morfológica, y presenta tendencia a la polisíntesis y a la aglutinación. Como es característico de las lenguas sintéticas, posee una rica morfología derivacional. En cuanto al orden de los constituyentes, el orden básico -aunque flexible- es AVO y VS. Con respecto al dominio nominal, la lengua distingue entre nombres alienables e inalienables, y exhibe marcación en el núcleo en las frases posesivas. El nombre puede presentar marcas morfológicas de poseedor, número y género. En esta lengua, el género es un rasgo léxico inherente por lo cual la oposición entre masculino y femenino se marca morfológicamente sobre unos pocos nombres, en particular los derivados. Los compuestos nominales constituyen construcciones indivisibles, poseen un orden fijo y funcionan como una unidad fonológica, morfológica y semántica. Como el resto de las lenguas guaycurúes, el toba presenta un sistema de seis clasificadores demostrativos que se anteponen a los nombres y que combinan rasgos configuracionales (forma y posición) con deixis (proximidad, movimiento y ausencia). Además de los clasificadores demostrativos, el sistema de género y los recursos de formación de léxico -como la derivación y la composición- contribuyen a la clasificación lingüística en esta lengua.

\footnotetext{
${ }^{7}$ De acuerdo a la Encuesta Complementaria de Pueblos Indígenas (ECPI 2004-2005) realizada por el INDEC-, de las 69.452 que se reconocen pertenecientes y/o descendientes en primera generación del pueblo toba, alrededor de la mitad (34.949 personas) habla y/o entiende la lengua indígena.
} 
Los datos del toba presentados en este artículo provienen del dialecto dapigemliek, hablado en el noroeste de la provincia del Chaco, y fueron recogidos personalmente en sucesivos trabajos de campo (2002 hasta la fecha) con hablantes residentes en el área chaqueña y en la localidad de Derqui (Provincia de Buenos Aires), en Argentina.

El trabajo se organiza de la siguiente manera. Las propiedades formales y funcionales de los términos de parentesco en la conformación de compuestos nominales se describen en la sección $\mathbf{2}$. Se discute asimismo la función de estos nombres de parentesco como términos de clase. En la sección 3., se indaga en las extensiones semánticas involucradas en el uso de los términos para 'padre' y 'madre' como parte de un incipiente proceso de gramaticalización. Las conclusiones, en 4., presentan una síntesis y, además, exploran los aportes de este estudio desde el punto de vista de las etnocategorizaciones y las relaciones sociales.

\section{Clasificación y composición: propiedades formales y funcionales}

Desde el punto de vista formal, los nombres relacionales de parentesco l-ta?a 'POS3-padre/ su padre' y l-ate?e 'Pos3-madre/ su madre' conforman compuestos nominales de tipo posesivo $(\mathrm{N}+$ Pos3-N) (Cúneo 2013a). Los términos documentados funcionan como núcleo sintáctico de la construcción compuesta y representan el término poseído (presentan la marca morfológica de posesión inalienable de $3^{a}$ persona $l$-). No obstante, el núcleo semántico del compuesto es el primer constituyente. Por ejemplo:

(4) poqo-latere

ortiga ${ }^{8}$-TC.FEM

'tipo de ortiga' (más peligrosa que poqo)

(5) qapoñi-lta?a

chanchita'9-TC.MASC

'tipo de pez' Cichlasoma sp. (de mayor tamaño que qapoñi)

La oposición lta?a (MASC)/ latePe (FEM) representa una distinción de género gramatical; ${ }^{10}$ estas formas presentan concordancia morfológica con el primer

\footnotetext{
${ }^{8}$ Urtica circularis.

${ }^{9}$ Refiere a un tipo de pez, Bujurquina vittata, Cichlasoma dimerus.

${ }^{10}$ No observamos, en principio, diferencias semánticas ni funcionales entre ambos términos, que puedan estar relacionadas con las funciones bioculturales del padre y de la madre. Un ejemplo contrario es el que describe Eberhard (2009:358-9) para la lengua mamaindê (familia nambikwara, Amazonia sur), donde la marca de género femenino -ta puede especificar el significado 'madre' así como el tamaño grande y la peligrosidad. El autor
} 
constituyente del compuesto. En (6) el nombre tePesaq determina el género masculino -propio de los nombres de abejas-, mientras que kotapik, en (7), es típicamente femenino -como todos los nombres para árboles. La concordancia de género se observa también en el determinante demostrativo.

(6) ñi te?esaq-lta?a

DD.MASC tipo.de.abeja.MASC-TC.MASC

'abeja casera' Apis mellifera (variedad europea, introducida)

(7) a-da kotapik-late?e

FEM-DD quebracho.colorado ${ }^{11}$.FEM-TC.FEM

'quebracho santiagueño' Schinopsis lorentzii

En un trabajo anterior (Cúneo 2013a), hemos argumentado que los términos de parentesco pueden ser considerados términos de clase tal como han sido definidos para las lenguas del este y del sudeste asiático. En estas lenguas, un término de clase (class term, Haas 1942; De Lancey 1986; Grinevald 2000; Beckwith 2007:140-156, 205-206) -también denominado nombre de clase (class noun, Bisang 1993, 1999; Aikhenvald 2000)- se define como el núcleo morfológico de un nombre compuesto, que es modificado por uno o más lexemas (o término calificador); se caracteriza por su generalidad semántica y configura una clase o categoría que muchas veces representa niveles taxonómicos mayores. Este tipo de compuestos permiten conformar, además, categorías relativamente abiertas.

Según Grinevald (2000:59-61), los términos de clase operan -como la morfología derivacional o la composición-al nivel de la palabra, y participan en la lexicogénesis de una lengua. Poseen un claro origen léxico y pueden ocurrir también como nombres independientes; sin embargo, han perdido en muchos casos su significado original. Además, han sido documentados especialmente en la conformación de campos léxicos específicos como el de la flora y la fauna (Aikhenvald 2000:87). Grinevald (2000) describe los términos de clase como un mecanismo de clasificación nominal de tipo más léxico que gramatical. ${ }^{12}$

relaciona esta asociación con el hecho de que, en muchas especies de la selva, la hembra es la más temida especialmente cuando cuida a su cría. Por ejemplo, tehãtatu 'la madre de todas las víboras/ una víbora grande/ la anaconda' (teha 'víbora') (359). Estos atributos semánticos y funcionales del género femenino no son compartidos en mamaindê por las formas masculinas.

${ }^{11}$ Schinopsis balansae.

${ }^{12}$ En su escala léxico-gramatical de sistemas de clasificación nominal, la autora ubica en el polo más léxico a los términos de clase y a los términos de medida. Los sistemas de género y clase nominal se ubican, por el contrario, en el polo más gramatical, en tanto que los clasificadores (nominales, numerales, posesivos, demostrativos) se encuentran en una posición intermedia (Grinevald 2000). 
Los términos de clase han sido documentados -como mencionamos- en lenguas del este y sudeste asiático (DeLancey 1986; Bisang 1999), como el japonés, el mandarín, el thai o el tibetano; en América, Facundes (2009) analiza el fenómeno en apurinã (familia arawak). Por ejemplo, en thai (Beckwith 2007:148), el término de clase yuu categoriza nombres de víboras: yuu khiaw 'víbora verde', yuu lyam 'serpiente pitón', yuu hàw 'cobra'. Otros términos de clase documentados en thai (DeLancey 1986:439) son los siguientes: khon 'persona', duay 'objeto redondo', lam 'objeto alargado'. ${ }^{13}$ Así también, en inglés (Grinevald 2000:59), el término tree conforma nombres de árboles, en tanto -berry especifica frutas (apple tree, banana tree, etc.; strawberry, blueberry, etc.). De la misma manera, el uso del término -man designa varios tipos de agentes: mailman, policeman, etc. No obstante, si bien es un fenómeno documentado en la bibliografía sobre clasificación nominal, no existe un acuerdo común sobre las propiedades que definen a los términos de clase en las lenguas del mundo. Debido a que estos han sido caracterizados según criterios específicos en las lenguas particulares, diferentes tipos de compuestos nominales fueron incluidos dentro del fenómeno de los términos de clase. Por este motivo, autores como Beckwith (2007:141) observan que se trata del tipo de sistema de clasificación nominal más "incomprendido" e "ignorado".

En toba, los términos late?e y lta?a poseen propiedades formales y funcionales que los acercan a los términos de clase, de acuerdo a la descripción anterior. ${ }^{14}$ Desde el punto de vista funcional, conforman clases relativamente abiertas de nombres dado que nuevos lexemas compuestos pueden formarse mediante este recurso. Poseen, por lo tanto, un uso creativo. No obstante, si bien los conjuntos de nombres compuestos conformados mediante términos de parentesco constituyen clases formales, no conforman clases semánticas consistentes y no están necesariamente involucrados en categorías taxonómicas, como observo más adelante (en 4.). Dado que participan activamente en la lexicogénesis de la lengua toba, permiten organizar campos semánticos y ampliar el léxico. Son recurrentes en los dominios semánticos que presentamos a continuación.

En primer lugar, late?e y lta?a contribuyen a conformar neologismos en

\footnotetext{
${ }^{13}$ DeLancey (1986) discute la estrecha relación entre términos de clase y clasificadores. De hecho, en las lenguas de la familia tai, los clasificadores tienen su origen en los términos de clase, y ambos sistemas de categorización nominal aún coexisten.

${ }^{14}$ En Cúneo (2013:256-294), proponemos que padre/ madre integran el siguiente conjunto de términos de clase utilizados en el vocabulario etnobiológico en toba: lyalek (MASC)/ lyale (FEM) 'cría'/‘tamaño pequeño'; liek (MASC)/ lashe (FEM) 'habitante', indica 'hábitat, ambiente' de una entidad biológica; lio '(animal) depredador'; lalaGat 'insecto, parásito, abeja, avispa'; lalaqte 'ave pequeña'; ala 'fruto'. No obstante, este tema necesita más estudio.
} 
diferentes dominios léxicos. Por ejemplo, conceptos introducidos a partir del contacto con la sociedad mayoritaria, como (8), o bien fenómenos ambientales destacados, como (9) y (10) (ejemplos extraídos de Cúneo 2013c):
(8) wataGanaq-lta?a
policía ${ }^{15}$-TC.MASC
'capitán, sargento'
(9) awot-lta?a
lluvia-TC.MASC
'diluvio'
(10) la?at-lta?a
viento-TC.MASC
'tornado'

En particular, los morfemas de padre y madre permiten especificar las partes del cuerpo humano, tanto exteriores (11) como interiores (12):
(11) l-qona-late?e
POS3-dedo-TC.FEM
'su dedo gordo'
(12) l-ailshik-lta?a
POS3-intestino-TC.MASC
'su intestino grueso'

De manera destacada, estos términos constituyen un recurso privilegiado en la formación de nombres del mundo natural así como en la identificación de variedades y especies. En los siguientes nombres compuestos, el término calificador (primer constituyente) representa un nombre genérico:
(13) Pawaq-pi-lta?a
pasto-PL-TC.MASC
'gramínea aromática' (de tamaño considerable) Cymbopogon citratus (Poa- ceae)
(14) Pele?-lta?a
loro-TC.MASC
'loro hablador' Ara sp.

Como adelantamos en la Introducción, los términos late?e y lta?a están atravesados por un fuerte significado social y de relevancia cultural, dado que denominan a los 'dueños', 'jefes' o 'padres' de las especies animales y

\footnotetext{
${ }^{15}$ Lit. 'el que espera' wataGan-aq 3.esperar-NMLZ.
} 
vegetales. ${ }^{16}$ Se trata de entidades no humanas que las dominan y protegen, con las que los humanos, especialmente los chamanes, mantienen relaciones. Para nombrar a los dueños, los términos de parentesco se añaden a los nombres de los animales que están bajo su dominio. Por ejemplo:

(15) wallikyaGay-lta?a

carpincho-TC.MASC

'dueño de los carpinchos'

(16) da?aylo?ok-lta?a

yacaré-TC.MASC

'dueño de los yacarés'

(17) ñi?imshi-late?e

guazuncho-TC.FEM

'dueña de los guazunchos'

Estos seres pueden tener corporalidad tanto humana como animal y cumplen funciones de protección del grupo de especies del que forman parte; tienen la facultad de aparecer y desaparecer de la vista de los humanos y transmitir mensajes; y "hay que respetar[los] en tanto líder[es] de un grupo de animales entendidos como importantes para la subsistencia de los qom" (Medrano 2012:239-240).

Con respecto al género gramatical, el nombre mantiene el género de la base semántica a la que se adjunta. En los ejemplos anteriores, da?ailo?ok lta?a es masculino ( $>$ da?ailo?ok MASC) mientras que ñi?imshi late?e es femenino ( $>$ niiPimshi FEM). A su vez, el género gramatical motiva la corporalización en hombre o mujer de cada uno de los dueños.

Si bien el tamaño puede constituir un rasgo para la identificación de estos "dueños", poseen otras cualidades que los caracterizan. En palabras de Valentín Suárez, maestro bilingüe y líder político qom (en Tola y Suárez 2013:60):

Decimos así porque al que llamamos [?adaGanaq 'víbora']-late' $e^{17}$ creemos que es una especie de víbora que es la mayor de las víboras y cuando aparece lo hace como ser humano, mujer, princesa, pero es una existente más antigua y más mayor que los humanos. Estaban ya ahí desde antes que los seres humanos. Como forma de respeto los

\footnotetext{
${ }^{16}$ Sobre el tema de los dueños de los animales y las plantas entre los tobas, cf. Miller 1979; Wright 1992, 2005; Tola 2010, entre otros. Existe abundante bibliografía sobre el tema en otros grupos indígenas tanto chaqueños como amazónicos; cf. Tola y Suárez (2013:59) y López (2013:118) para una mención de la literatura pertinente.

${ }^{17}$ El sonido oclusivo glotal / $/$ en late?e es representado ortográficamente mediante un apóstrofo (').
} 
llamamos así porque son mayores. Para su especie son -late'e y -lta'a porque son más grandes de tamaño y de todo (fuerza, potencia, color), pero para los humanos son mayores de existencia (de edad).

En un trabajo entre los mocovíes, Alejandro López (2013:120) sintetiza los atributos de los dueños de las especies de la siguiente manera:

"La interacción con estos seres poderosos es imprescindible para los humanos, ya que los dueños son la fuente de los recursos de los cuales depende la vida humana. Esta interacción debe estar regulada porque los dueños son excesivos, anómalos y están fuera de la sociabilidad humana ordinaria." [El subrayado es mío.]

Como observamos hasta aquí, los lexemas de padre y madre en toba no poseen un significado homogéneo. Argumentaré en la siguiente sección (3.) que ambas cualidades mencionadas -"exceso" y "anomalía"- están implicadas también en las extensiones semánticas de los términos de parentesco en toba.

\section{Extensión semántica y gramaticalización}

A broad, composite definition of this Arabic morpheme [of 'mother'] would then be something like: 'mother; source, origin; basis, foundation; original, original version; the gist, the essence; (as attribute) original, primary, basic, parent; main or chief part of a thing, or that which is a compriser or comprehender of things' (Matisoff 1992:296)

En la sección previa, hemos mostrado que los términos para madre y padre en toba son elementos recurrentes en la formación de nombres compuestos de manera productiva y que poseen un rol destacado en el léxico de la lengua.

Desde el punto de vista semántico, se observa un desplazamiento conceptual. Si bien los términos nucleares de parentesco pueden ser utilizados frecuentemente para referirse a su significado original (literal), adoptan en otros casos un significado más general y funcional. En este sentido, experimentan cierto grado de erosión del significado, adquiriendo propiedades semánticas más abstractas, menos concretas. Los significados que coexisten pueden explicarse a partir de extensiones metafóricas de los conceptos de parentesco. Así, el significado fuente de 'padre' y 'madre' se preserva cuando las formas se usan como ítems léxicos independientes. Cuando forman parte de compuestos nominales, en cambio, su significado se vuelve más abstracto y genérico. En estos casos, se adjuntan de manera productiva a diferentes nombres para expresar nociones de aumentatividad, semejanza y no-prototipicalidad, dando lugar a términos de clase. 
De esta manera, el principal argumento que distingue en toba los compuestos conformados mediante términos de clase de los compuestos posesivos prototípicos es que aquellos participan de incipientes procesos de gramaticalización. Se trata de un proceso gradual, que involucra también fusión morfológica y coalescencia fonológica (por ejemplo, tesaq-lta?a $>$ tesa Galta?a 'variedad de abeja' cf. ejemplo (6)), así como también un reanálisis del núcleo de la construcción hacia término de clase: ${ }^{18}$

$$
\text { (18) }[\mathrm{N}+\mathrm{N}[\text { núcleo morfológico }] \text { FN } \quad>\quad[\mathrm{N}-\mathrm{TC}] \text { COMP }
$$

La composición ha sido considerada una fuente histórica destacada de diferentes sistemas de clasificación. ${ }^{19}$ En particular, el desarrollo de clasificadores a partir de términos de clase que forman compuestos nominales ha sido ampliamente documentado (cf. Bisang 2011 para el desarrollo de este tópico). Sin embargo, la diferencia entre morfemas de derivación y términos de clase usados en procesos de composición no está siempre resuelta, y ambos han sido llamados clasificadores (Grinevald 2000:59-60).

En relación con las extensiones metafóricas y los procesos de gramaticalización de los morfemas que significan 'madre', Matisoff (1992) investiga los mothermorphs tanto en inglés como en las lenguas del este y del sudeste asiático, y arriba a ciertas generalizaciones translingüísticas acerca de la evolución metafórica y la gramaticalización de estos morfemas. El autor presenta estas tendencias de la siguiente manera (Matisoff 1992:333):

(19) mother

1. mother (literal female parent)

2. female humans in general and/or female of animals

3a. origin/source/matrix ("ORIGINATIVE") and/or

3b. big/main/most important (AUGMENTATIVE)

4. object in general; noun marker (bulk-providing marker for nounroots); nominalizer of verbroots

(20) father

1. father (literal male parent)

2. male person in general; male in general

3. person in general

4. object in general; bulk-provider for nounroots; nominalizer of verbs

\footnotetext{
${ }^{18}$ Cf. Epps (2009) para un análisis detallado del incipiente proceso de gramaticalización del sistema de clasificación nominal en hup (lengua amazónica).

19 Los clasificadores numerales de las lenguas del sudeste asiático (Bisang 1999) y los clasificadores nominales de las lenguas mesoamericanas (jacalteco, Craig 1986) y amazónicas (hup, Epps 2007) consituyen sistemas de clasificación que se originan en la composición nominal.
} 
En este mismo sentido, Heine y Kuteva (2002:209) observan que se trata de "a process whereby human nouns (child, mother, father, woman, man), on account of some salient semantic characteristics, give rise to grammatical markers highlighting that characteristic". Si bien el vínculo entre la expresión de la relación parental y la emergencia de significados de diminutivo es un fenómeno ampliamente documentado (Heine y Kuteva 2002; Grandi 2011; Cúneo 2013b para el caso del toba); la relación entre los términos de parentesco 'madre' / 'padre' y la noción de aumentativo ha recibido, no obstante, menor atención. En kana (una lengua de la familia Niger-Congo hablada en el sudeste de Nigeria), por ejemplo, el prefijo de aumentativo $k a ́$ - deriva probablemente de la palabra para madre a través de un proceso mediante el cual morfemas léxicos, especialmente aquellos que conforman compuestos, devienen afijos de derivación (Ikoro 1996:61, en Aikhenvald 2007:58). De la misma manera, algunas lenguas tibeto-burmanas también gramaticalizan las relaciones sociales básicas para funcionar como clasificadores nominales, y un ejemplo de esta ruta de gramaticalización es el uso de los términos madre e hijo para funcionar como aumentativo y diminutivo (cf. Matisoff 1992).

Proponemos que la lengua toba gramaticaliza las relaciones sociales parentales (madre, padre) para funcionar como mecanismos de clasificación. El uso documentado de los términos nucleares de parentesco en las denominaciones vernáculas sugiere una función en tanto mecanismo de categorización nominal que codifica significados cercanos a la noción de semejanza y no-prototipicalidad junto a la de aumentativo.

Un extenso grupo de nombres derivados mediante estos términos categorizan en toba entidades tanto zoológicas como botánicas, y su función es distinguir especies que son de alguna manera semejantes. Se establece una relación de semejanza con relación a una entidad concebida como prototípica. En algunos casos, opera el rasgo semántico de tamaño, pero la analogía puede construirse también en base a otras propiedades salientes, o bien distinguirse del prototipo ligeramente en cuanto a comportamiento o hábitat. No obstante, la relación semántica que se establece entre el primer término y el nombre compuesto no es de tipo taxonómico o jerárquica, en la que el nombre representa una subclase. ${ }^{20}$ Los ejemplos presentan nombres de aves (21)-(22), víboras (23) y plantas (24)-(25)-(26).
(21) qoto?-lta?a
paloma ${ }^{21}$-TC.MASC
‘tucán' Ramphastos discolorus y R. toco

\footnotetext{
${ }^{20}$ Se necesita un estudio etnobiológico que confirme estas afirmaciones.

${ }^{21}$ Columba sp.
} 
(22) chochon-late?e

carpintero ${ }^{22}$-TC.FEM

'carpintero real' Chrysoptilus melanochlorus

(23) masalaGa-late?e

yarará-TC.FEM

'musurana' Clelia clelia

(24) waloq-lta?a

algodón-TC.MASC

'algodón del monte' Cienfuegosia drummondii

Presenta similitudes morfológicas con los frutos del algodón (Martínez y

Cúneo 2009).

(25) chayk-late?e

palmera-TC.FEM

'yuca, variedad de palma'

(26) ladanka-late?e

naranja-TC.FEM

'naranjillo' Fagara naranjillo

Sus hojas poseen un intenso aroma similar al de la naranja (Martínez y Cúneo 2009).

En relación con su función aumentativa, ${ }^{23}$ los términos de parentesco nombran especies de alguna manera destacadas para el hombre, por ejemplo, más peligrosas, como la víbora coral que es venenosa y mortal (cf. nota al pie 10 para un ejemplo análogo en mamaindê).

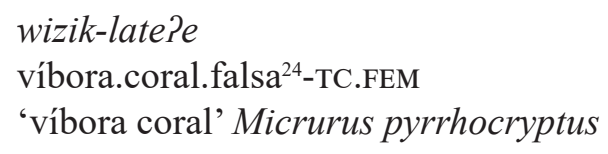

wizik-late?e

víbora.coral.falsa ${ }^{24}$-TC.FEM

'víbora coral' Micrurus pyrrhocryptus

Cuando su función de no-prototipicalidad se encuentra en foco, los nombres compuestos con estos términos de clase refieren a especies menos abundantes (Gustavo Martínez c.p.) como el quebracho santiagueño Schinopsis lorentzii (ejemplo (7)) o introducidas recientemente en la cultura como la abeja casera Apis mellifica (ejemplo (6)), la oveja o el banano:

\footnotetext{
${ }^{22}$ Se refiere especialmente al 'pájaro carpintero campestre' Colaptes campestris.

${ }^{23}$ La lengua posee otros recursos para la función típica de aumentativo (mayor tamaño o dimensión). Entre ellos, los atributivos derivados de los términos de parentesco (ltaPadayk 'grande (MASC)' y late?eday 'grande (FEM)'). Cf. Cúneo (2013c) para profundizar el contraste entre el uso de términos de parentesco y otros recursos léxicos y gramaticales.

${ }^{24}$ Oxyrhopus guibei, O. rhombifer rhombifer.
} 
(28)

qageta-late?e

guasuncho-TC.FEM

'oveja' 25

(29) perelak-late?e

achira $^{26}$-TC.FEM

'banano'

En síntesis, una definición amplia de los morfemas 'madre' y 'padre' en toba puede comprender los siguientes significados:

\section{(30) late?e (FEM) / Ita?a (MASC)}

1. madre / padre

2. grande (tamaño) / rasgo destacado (color, olor, comportamiento, hábitat) (aumentativo)

3. semejante, no prototípico (clasificador: tipo similar)

4. dueña/o de especie biológica

Notamos que los primeros significados $1>2$ reflejan una tendencia metafórica universal, en consonancia con los hallazgos de Matisoff (1992). No obstante, los significados 3 y 4 parecen componer desarrollos propios del toba. ${ }^{27}$ Destacamos también que el significado 4 'dueña/o' de las especies de plantas y animales combina las nociones semánticas de intensidad o aumentativo y aquellas de no prototipicalidad, como leíamos en la cita de López (cf. arriba) con respecto a las cualidades de "exceso" y "anomalía".

En cuanto a la oposición late?e 'madre/ lta?a 'padre', esta distingue metafóricamente género gramatical femenino y masculino. Ambas formas comparten, por lo tanto, los mismos rasgos semánticos.

\footnotetext{
${ }^{25}$ El nombre actual para guasuncho (Mazama sp.) es ñi?imshi, mientras que qageta se conserva para oveja. Agradezco a Celeste Medrano por iluminarme sobre estos ejemplos.

${ }^{26}$ Canna glauca.

27 En Messineo y Cúneo (2011), describimos que la conexión entre los términos 'padre'/'madre' y la noción de aumentativo puede considerarse un procedimiento común a las lenguas de la familia guaycurú. La relación con las nociones de 'raro', 'extraño', 'defectuoso' o 'desusado' en nombres de plantas y de animales se registra también en un estudio etnobiológico entre los tobas del oeste (Arenas y Porini 2009:127-9). Entre las lenguas mataguayas, cf. Scarpa $(2010: 173,180)$ con referencia al vocabulario botánico chorote conformado mediante los términos t'iój / tóik que señalan 'falso, deficiente o deforme' en relación a un genérico que funciona como prototipo; también se utilizan para nombrar plantas introducidas y, en menor medida, cultígenos en relación a su semejanza con plantas silvestres. Más allá de la región chaqueña, recursos similares para categorizar variedades o especies 'no autóctonas', 'menos conocidas' o 'falsas' se documentan, por ejemplo, en la lengua ka’apor (tupí-guaraní, Balée 1989:17) y en tzeltal (maya, Berlin et al. 1973, 1974).
} 
Finalmente, si entendemos que una de las funciones destacadas de los sistemas de clasificación en el dominio nominal es la identificación (Bisang 2011:19) podemos explicar el surgimiento de este mecanismo de categorización. En términos de Bisang (2011:19):

In the case of identification, classification is employed to narrow down the search space for finding an object in space. A particular sensory perception and its properties are compared to the properties of other sensory perceptions in order to identify that particular perception by subsuming it under a certain concept and thus distinguishing it from other concepts that are equally present within the speech situation in which a given utterance is made. Both functions [individuation and identification] set the pragmatic stage for processes of grammaticalization which end up with the following grammatical functions and categories.

\section{Conclusiones y perspectivas}

Este artículo profundizó e integró estudios anteriores con el fin de precisar los aspectos formales, semánticos y funcionales de los morfemas léxicos 'madre' late?e y 'padre' lta?a en toba. Desde el punto de vista formal, estos conforman compuestos posesivos que, de manera productiva, permiten ampliar y organizar el vocabulario de la lengua, predominantemente en los dominios semánticos de las plantas y los animales y, en menor medida, en las partes del cuerpo y otros neologismos. Hemos detallado las nociones semánticas asociadas a estos lexemas que, más allá de su significado literal que refiere a los lazos parentales, poseen significados ligados al aumentativo -en consonancia con los hallazgos tipológicos en otras lenguas-, y combinan también las nociones de aproximación o semejanza y de no-prototipicalidad -rasgo tipológicamente inusual. Así también, los términos de parentesco permiten delinear la clase de los "dueños de las especies", entidades que interactúan cotidianamente con los qom. Su función específica en la categorización de nombres los acerca a los términos de clase y participan de esta manera de incipientes procesos de gramaticalización de acuerdo a las siguientes propiedades. En primer lugar, pierden en parte su significado lexical para adoptar significados más generales y abstractos; exhiben fusión fonológica y concordancia morfológica de género con el primer constituyente del compuesto. A su vez, un leve reanálisis de las formas permite que estas participen de la función de la clasificación nominal en dominios léxicos específicos. Asimismo, se destacan por la frecuencia y la productividad, dado que nuevas formas utilizan el mismo procedimiento. Desde el punto de vista pragmático, contribuyen a la especificación e identificación de entidades. 
Los datos y el análisis presentados permiten revisar las relaciones entre clasificación, derivación y lexicogénesis. Se trata de un procedimiento particularmente rico; futuros estudios podrán observar si podría ser considerado como un fenómeno areal chaqueño.

\section{1 ¿Categorización no taxonómica?}

Con el fin de explicar la función de los términos late?e y lta?a en la categorización de nombres y en la codificación de categorías nativas en toba, resulta particularmente interesante la idea propuesta por Scarpa (2010:172-3) para su análisis de ciertos términos etnobotánicos chorote (mataguaya). El autor propone que los términos de este tipo no denotan una relación jerárquica con el genérico al cual acompañan ni denominan una categoría subgenérica (especie, variedad), sino que forman clases que poseen más bien un 'aire de familia'. El filósofo austríaco Ludwig Wittgenstein utiliza la metáfora de 'aire de familia' [family resemblance] para describir la estructura semántica de las palabras (en particular la estructura de la categoría definida por la palabra Spiel 'juego'). Una categoría no puede definirse, según Wittgenstein, a partir de un conjunto delimitado, específico y absoluto de propiedades comunes sino que se explica a partir de una red de similitudes superpuestas y entrelazadas, así como los miembros de una familia se relacionan según su contextura física, sus rasgos, su color de ojos, su forma de caminar, su temperamento, etc. (Wittgenstein 1978:31-3 en Taylor 1989:38-40). Algunos miembros comparten algunos de estos atributos, otros miembros comparten otros, pero no hay atributos comunes a todos los miembros. ${ }^{28}$

En este sentido, aunque con el interés en la búsqueda de universales semánticos, Wierzbicka (1992:4) distingue los conceptos -probablemente universales- de kind (tipo) y like (similaridad). ${ }^{29}$ Mientras el primero es taxonómico ( $\mathrm{X}$ is a kind of $\mathrm{Y}$ ), el segundo no lo es (X is like $\mathrm{Y}$ ). La noción no taxonómica de similaridad o like permite caracterizar mejor la función de los términos de parentesco estudiados, dado que la relación semántica que se establece entre un término clasificador y el nombre al que clasifica no es siempre de tipo taxonómico, es decir, jerárquica o de inclusión.

\footnotetext{
${ }^{28} \mathrm{cf}$. Harrison (2007:45-8) para la explicación de un procedimiento semejante ejemplificado en los nombres de aves en la lengua wayampi hablada en la Amazonia brasileña. El autor utiliza las nociones de 'jefe' y 'seguidores' [chief and followers] para analizar los agrupamientos de entidades en torno a un prototipo o 'jefe' y sus 'seguidores'. Los agrupamientos se basan en criterios morfológicos (apariencia), ecológicos (hábitat) y etológicos (comportamiento) de los organismos juzgados como relevantes por los hablantes. El conocimiento sobre estas categorizaciones se codifica en los nombres en la lengua wayampi.

29 "To put it in terms of the theory of universal semantic primitives, the notion of kind (" $\mathrm{X}$ is a kind of Y") cannot be reduced to the notion of like ("X is like Y")" (Wierzbicka 1992:4).
} 
De la misma manera, los términos late?e y lta?a poseen la función de denominar una entidad 'familiar', 'relacionada', 'semejante', y, de manera general, permiten configurar relaciones semánticas no necesariamente taxonómicas (jerárquicas) sino relacionales - idea que introduje en Cúneo (2013c). El nombre compuesto conformado mediante este término puede compartir con el prototipo rasgos semejantes en cuanto a su forma y distinguirse en otros aspectos. Finalmente, a esta noción de semejanza, se añade la noción de aumentativo (en términos de tamaño, potencia, color, peligrosidad, exotismo). En este sentido, estos términos combinan una función clasificatoria (semejanza y no prototipicalidad) y una aumentativa.

Si bien se necesita un estudio sobre etnocategorizaciones para arrojar luz con mayor certeza sobre esta cuestión, estas conclusiones permiten un acercamiento a las relaciones entre entidades definidas sobre la base de un prototipo $\mathrm{y}$, en particular, a los vínculos semánticos relacionales, en contraste con aquellos basados en jerarquías.

\subsection{Vínculos metafóricos de maternidad y paternidad}

Por último, desde una perspectiva etnográfica, el análisis que presentamos puede contribuir a pensar en la extensión metafórica de los vínculos de maternidad y paternidad. En este sentido, con el objetivo de explicar diversos sistemas de clasificación en las lenguas del mundo, se ha prestado atención a la forma en la que los humanos interactúan con el medio y entre sí. A partir de su pionero artículo "What Are Classifiers Good for?", Denny (1976) propone que las categorías marcadas por los sistemas de clasificación lingüística se basan en la interacción que los hablantes tienen con los objetos y los seres de su entorno. Denny distingue tres grandes tipos de interacción: interacción social (los humanos son categorizados de acuerdo al sexo, la edad, el estatus social), física (que clasifica los objetos según su forma, consistencia y material) y funcional (según el uso o función sociocultural). Los criterios semánticos propuestos por Denny constituyeron la base para el estudio de los sistemas de clasificación en las lenguas particulares.

En consonancia con la propuesta de Denny, nos preguntamos si los morfemas de 'madre' y 'padre' en toba con los diversos significados asociados y sus funciones en la clasificación nominal emergerían a partir de la manera en la que se definen y se configuran las relaciones sociales parentales. En un estudio antropológico sobre las terminologías de parentesco entre los qom, Tola y Salamanca (2009) observan que tanto el sistema de actitudes como las designaciones tobas son los que permiten que los parientes afines (no consanguíneos) sean llamados y tratados como los propios consanguíneos. De 
esta manera, el uso de las denominaciones late?e y lta?a permitiría configurar vínculos de cercanía afectiva, de "familiaridad". En un trabajo reciente, Tola y Suarez (2013:59) se interrogan si es posible pensar que las relaciones entre seres humanos y dueños sean concebidas en términos semejantes a los lazos de parentesco. Los autores indagan en la forma de vincularse, a través de la compasión, entre padres e hijos, entre dueños y animales. En este marco, el presente análisis estimula un estudio más profundo para indagar si la forma en la que se definen socialmente las relaciones late?e y lta?a entre los seres cobra importancia para definir los significados lingüísticos, y viceversa.

Finalmente, destacamos que, con respecto a la propuesta tipológica de Denny (1976) -que distingue sistemas de clasificación según los tipos de interacción social, física o funcional, pero no los tres a la vez- la lengua toba plasma en el uso y la función de los términos de parentesco estudiados una relación de continuidad entre el mundo de los seres humanos y el mundo físico.

\section{Referencias}

Aikhenvald, Alexandra. 2000. Classifiers. A Typology of Noun Categorization Devices. Oxford: Oxford University Press.

Aikhenvald, Alexandra. 2007. "Typological distinctions in word-formation." En Language Typology and Syntactic Description, ed. Timothy Shopen, vol. III: Grammatical Categories and the Lexicon, 1-65. Cambridge: Cambridge University Press. $2^{\text {nd }} e d$.

Allan, Keith. 1977. "Classifiers." Language 53:285-311.

Arenas, Pastor y Gustavo Porini. 2009. Las aves en la vida de los tobas del oeste de la Provincia de Formosa. Asunción: Editorial Tiempo de Historia.

Balée, William. 1989. "Nomenclatural patterns in Ka'apor ethnobotany." Journal of Ethnobiology 9, 1:1-24.

Beckwith, Christopher. 2007. Phoronyms. Classifiers, Class Nouns, and Pseudopartitive Construction. New York: Peter Lang Publishing.

Berlin, Brent, Dennis E. Breedlove y Peter H. Raven. 1973. "General Principles of Classification and Nomenclature in Folk Biology." American Anthropologist 75, 1:214-42.

Berlin, Brent, Dennis E. Breedlove y Peter H. Raven. 1974. Principles of Tzeltal plant classification. An introduction to the botanical ethnography of a Mayanspeaking people of Highland Chiapas. New York/ London: Academic Press.

Bisang, Walter. 1999. "Classifiers in East and Southeast Asian languages: Counting and beyond." En Numeral types and changes worldwide, ed. Jadranka Gvozdanovic, 113-85. Berlin: Mouton de Gruyter. 
Bisang, Walter. 2011. "Nominal and verbal classification - why the former is far more widespread than the latter." Paper presented at the Association of Linguistic Typology 9, Hong Kong, July 21-24.

Buckwalter, Alberto. 2001 [1980]. Vocabulario toba. Formosa/ Indiana: Equipo Menonita/ Mennonite Board of Missions. Ed. Revisada.

Censabella, Marisa. 2002. "Descripción funcional de un corpus en lengua toba (Familia Guaycurú, Argentina). Sistema fonológico, clases sintácticas y derivación. Aspectos de sincronía dinámica.” PhD diss., Universidad Nacional de Córdoba.

Craig, Colette. 1986. "Jacaltec noun classifiers. A study in grammaticalization." Lingua 70:241-284.

Cúneo, Paola. 2012. "Términos especializados en la composición de nombres etnobiológicos en toba (guaycurú)." Revista UniverSOS, Revista de Lenguas Indigenas y Universos Culturales 9:133-46. Universitat de València.

Cúneo, Paola. 2013a. Formación de palabras y clasificación nominal en el léxico etnobiológico en toba (guaycurú). Lincom Studies in Native American Linguistics 68. Munich: Lincom Europa.

Cúneo, Paola. 2013b. "Diminutivo en toba (guaycurú): forma y función.” Ponencia presentada en el III Encuentro de Lenguas Indígenas Americanas. Universidad Nacional de Río Negro, San Carlos de Bariloche, 15 al 17 de mayo.

Cúneo, Paola. 2013c. "Augmentative in Toba (Guaycuruan): Form and Function." En Word formation in South American languages, eds. Swintha Danielsen, Katja Hannß y Fernando Zúñiga, 52-77. Studies in Language Companion Series 163, John Benjamins.

DeLancey, Scott. 1986. "Towards a history of Tai classifier systems." En Noun Classes and Categorization, ed. Colette Craig, 437-452. Amsterdam/ Philadelphia: John Benjamins.

Denny, Peter. 1976. "What are noun classifiers good for?" Papers from the 12th Regional Meeting of the Chicago Linguistics Society, 122-132.

Drayson, Nicholas. 2009. "Niwak Samtis. Diccionario Iyojwa'ja 'Lij - Kilay 'Lij (Chorote - Castellano)." En Hacia una nueva carta étnica del Gran Chaco VIII, eds. José Braunstein y Cristina Messineo, 91-174. Buenos Aires/ Las Lomitas: Centro del Hombre Antiguo Chaqueño.

Eberhard, David. 2009. Mamaindê Grammar: A Northern Nambikwara language and its cultural context. The Netherlands: Lot.

Epps, Patience. 2009. "O nascimento de um sistema de classificacao nominal." ReVEL. Edicao especial No 3. www.revel.inf.br.

Facundes, Sidi. 2009. "Productive compounding and noun classification systems: A case study in Apurina (Arawak)." ReVEL. Edicao especial No 3. www.revel. inf.br. 
Facundes, Sidney. 1994. "Noun Categorization in Apurina (Maipuran)." MA. Thesis, University of Oregon.

Grandi, Nicola. 2011. "Renewal and innovation in the emergence of Indo-European evaluative morphology." En Lexis 6: Diminutives and Augmentatives in the Languages of the World, eds. Lívia Körtvélyessy y Pavel Štekauer, 5-25.

Grinevald, Colette. 2000. “A morphosyntactic typology of classifiers.” En Systems of Nominal Classification, ed. Gunter Senft, 93-113. Cambridge: Cambridge University Press.

Haas, Mary. 1942. “The use of numeral classifiers in Thai.” Language 18:201-205.

Harrison, K. David. 2007. "An Extintion of (Ideas about) Species." When Languages Die. The Extinction of the World's Languages and the Erosion of Human Knowledge, 23-5. New York: Oxford University Press.

Heine, Bernd y Kuteva, Tania. 2002. World Lexicon of Grammaticalization. Cambridge: Cambridge University Press.

Ikoro, Suanu M. 1996. The Kana Language. Leiden: University of Leiden.

INDEC - Instituto Nacional de Estadísticas y Censos, ECPI - Encuesta Complementaria de Pueblos Indígenas, 2004-2005. http://www.indec.gov.ar/ webcenso/ECPI/index_ecpi.asp. Acceso 31 de agosto, 2013.

López, Alejandro. 2013. "Las texturas del cielo. Una aproximación a las tipologías moqoit del poder." En Gran Chaco. Ontologías, poder, afectividad, eds. Florencia Tola, Celeste Medrano y Lorena Cardín, 103-131. Buenos Aires: Asociación Civil Rumbo Sur.

Martínez, Gustavo. 2009. "Fitonimia de los tobas bermejeños (Chaco Central, Argentina)." En Hacia una nueva carta étnica del Gran Chaco VIII, eds. José Braunstein y Cristina Messineo, 194-212. Buenos Aires/ Las Lomitas: Centro del Hombre Antiguo Chaqueño.

Martínez, Gustavo y Paola Cúneo. 2009. "Las denominaciones vernáculas y el conocimiento toba del entorno vegetal." Revista de Dialectología y Tradiciones Populares (RDTP), vol. LXIV, 2:149-68. Madrid: Instituto de Lengua Literatura y Antropología, Consejo Superior de Investigaciones Científicas.

Matisoff, James. 1992. "The Mother of All Morphemes: Augmentatives and diminutives in areal and universal perspective." Papers from the First Annual Meeting of the Southeast Asian Linguistics Society, eds. Martha Ratliff y Eric Schiller, 293-349. Arizona State University, Program for Southeast Asian Studies.

Medrano, Celeste. 2012. "Zoo-sociología qom: de cómo los tobas y los animales trazan sus relaciones en el Gran Chaco." PhD diss., Universidad de Buenos Aires.

Medrano, Celeste, Mauricio Maidana y Cirilo Gómez. 2011. Zoología Qom. Conocimientos tobas sobre el mundo animal. Santa Fe, Argentina: Ediciones Biológica. Serie Naturaleza, Conservación y Sociedad $N^{\circ} 3$. 
Messineo, Cristina. 2003. Lengua toba (guaycurú). Aspectos gramaticales y discursivos. Lincom Studies in Native American Linguistics 48. Munich: Lincom Europa.

Messineo, Cristina y Paola Cúneo. 2011. "Ethnobiological classification in two indigenous languages of the Gran Chaco Region: Toba (Guaycuruan) and Maká (Mataco-Mataguayan)." Anthropological Linguistics 53, 2:132-69.

Miller, Elmer. 1979. Los tobas argentinos. Armonía y disonancia en una sociedad. México: Siglo XXI.

Scarpa, Gustavo. 2010. "Hacia una etnotaxonomía vegetal chorote II: Clasificación de las plantas entre las parcialidades iyojwá'ja y iyowújwa del Chaco argentino." En Cristina Messineo, Gustavo F. Scarpa y Florencia Tola (comp.). Léxico y categorización etnobiológica en grupos indígenas del Gran Chaco, 157-198. Santa Rosa: Universidad Nacional de La Pampa.

Taylor, John. 1989. Linguistic categorization. Prototypes in linguistic theory. Oxford: Clarendon Press.

Tola, Florencia y Valentín Suarez. 2013. "Diálogo sobre los existentes de un entorno superpoblado en el contexto de la marisca y la reivindicación política del territorio." En Gran Chaco. Ontologías, poder, afectividad, ed. Florencia Tola, Celeste Medrano y Lorena Cardín, 45-76. Buenos Aires: Asociación Civil Rumbo Sur.

Tola, Florencia. 2010. "Maîtres, chamanes et amants." En Ateliers du LESC (Société d'Ethnologie de Nanterre) 34, comp. Valentina Vapnarsky y Aurore Monod Becquelin. http://ateliers.revues.org/8538.

Tola, Florencia y Carlos Salamanca. 2009. "Una revisión de las terminologías de parentesco y del régimen de alianza matrimonial entre los toba (qom) del chaco argentino." En VI Congreso Argentino de Americanistas 2008, 3:379393, coord. E. Cordeu. Buenos Aires: Sociedad Argentina de Americanistas y Dunken.

Wierzbicka, Anna. 1992. "What is a Life Form? Conceptual Issues in Ethnobiology." Journal of Linguistic Anthropology 2, 1:3-29.

Wittgenstein, Ludwig. 1978. Philosophical Investigations. Translated by G. E. M. Anscombe. Oxford: Basil Blackwell.

Wright, Pablo. 1992. "Dream, shamanism, and power among the toba of Formosa Province." En Portal of Power. Shamanism in South America, eds. Jean Langdon y Gerard Baen, 149-172. Albuquerque: University of New México Press.

Wright, Pablo. 2005. “Cosmografías.” Etnografías Contemporáneas 1, 1:173-210.

Data recebimento: 14/03/2014.

Data aceite: 18/04/2014. 